\title{
Publisher Correction: Growing Asian research networks
}

Monica Contestabile

Correction to: Nature Sustainability https://doi.org/10.1038/s41893-019-0232-3, published online 11 February 2019.

In the version of this Q\&A originally published, the founder of OJERI was mistakenly given as 'Kim Jung-Kyu'. The name should have read 'Kim Jeong-Gyu'. This has now been corrected. 